\title{
Multivariate cube integrated retinal variable to visually represent multivariable data
}

\author{
H.T. Nguyen ${ }^{1, *}$, A.V.T. Tran ${ }^{2}$, T.A.T. Nguyen $^{3}$, L.T. Vo ${ }^{4}$, and P.V. Tran ${ }^{5}$ \\ ${ }^{1}$ Rubber Industrial College, Binhphuoc, Vietnam, hongnguyen1611@gmail.com \\ ${ }^{2}$ Hochiminh City Economics College, Hochiminh City, Vietnam, ttvanh26@gmail.com \\ ${ }^{3}$ Thudaumot University (TDMU), Binhduong, Vietnam, tuyetnta@tdmu.edu.vn \\ ${ }^{4}$ Eastern International University, Binhduong, Vietnam, luc.vo@eiu.edu.vn \\ ${ }^{5}$ Thudaumot University (TDMU), Binhduong, Vietnam, phuoctv@tdmu.edu.vn ; phuoc.gis@gmail.com
}

\section{Abstract}

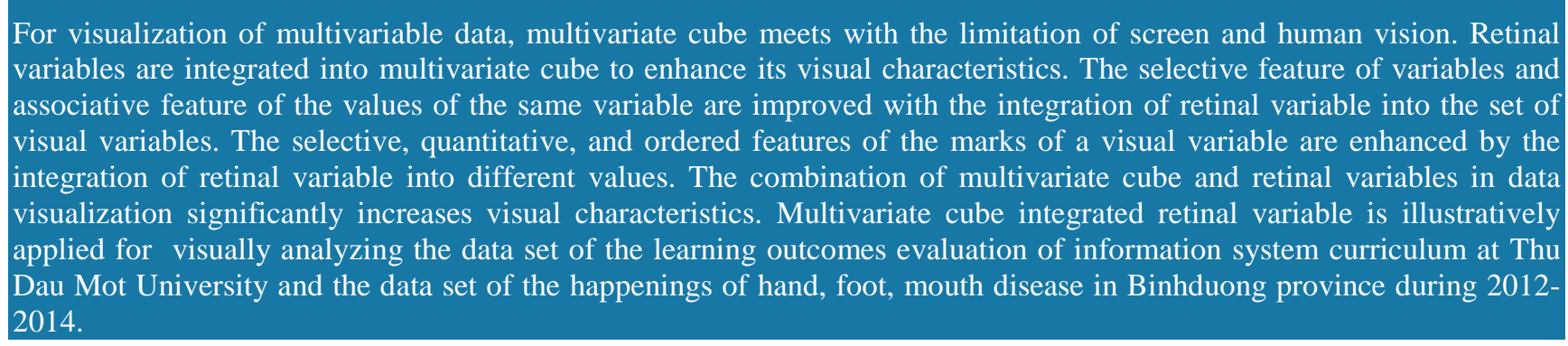

Keywords: visualization, multivariate cube, visual characteristic.

Received on 05 May 2017, accepted on 17 June 2017, published on 06 July 2017

Copyright (C 2017 H.T. Nguyen, licensed to EAI. This is an open access article distributed under the terms of the Creative Commons Attribution licence (http://creativecommons.org/licenses/by/3.0/), which permits unlimited use, distribution and reproduction in any medium so long as the original work is properly cited.

doi: 10.4108/eai.6-7-2017.152757

\section{Introduction}

Data visualization is a mapping of the significance implicit in data into human awareness. It converts data into human knowledge. In that, data set is represented as graph which people may easily perceive by their vision. With available experience and knowledge, data analysts may discover new knowledge from graphs representing data. In other words, the method of visual analysis enables analysts to extract information and discover new knowledge through their visional perception in viewing the graph visualizing data set. The worth of the acquired knowledge depends on the human capacity perceiving visual graph. The graphs of highly visual characteristics provide viewer with specific aspects of data significance. For multivariable data, data analysts are difficult to

*Corresponding author. Email:author@emailaddress.com understand visual graphs because of lowly visual characteristics of the graphs. Accordingly, visual characteristics of visual graphs play an important role in visual analysis.

The main idea to enhance visual characteristics of visual graphs is to integrate retinal variables into visual variables. Retinal variables improve visual characteristics of graphs according to the way user likes in order to increase the human perception of vision. The integration of retinal variables into visual variables enhances the selective feature of different visual variables and the associative feature of the marks representing values of the same variable. Retinal variables also stand out particular marks representing highest or lowest values of a data variable. In addition, soft tools of graphic technique enable analysts to interact directly with visual graphs to display various graph styles on screen. Viewing the whole 
graph representing a data variable and studying the correlation among graphs representing several data variables, analysts may extract information implicit in data. Utilizing available knowledge, data analysts may discover new knowledge or law from data set by viewing visual graphs representing data set from different directions of view. This article systemizes visual characteristics based on the human capacity of graphical perception of vision and the methods integrating retinal variables into graphs for data visualization.

The article is structured as follows. The next section summarizes of the human capacity of visional perception and visual characteristics necessary for the perception of vision. The third section presents the approaches to integrating retinal variables into multivariate cube to improve visual characteristics of graphs representing data set. In the fourth section, multivariate cube integrated retinal variables is applied to represent multivariable data of two case studies, the data of the estimation of higher education state of Thu Dau Mot University (TDMU), the data concerning the disease of hand, foot, and mouth in Binhduong province. The visual characteristics of graphs representing the two data sets are improved by the integration of retinal variables. The last section relates to the effectiveness of multivariate cube integrated retinal variables.

\section{The characteristics of visualization}

\section{2.1. The visional perception}

People acquire information through five senses, vision, hearing, touch, taste, and smell. As sensors, the sense organs collect information and excite human brain to transfer it to mind. For the five senses, vision is the sense which stimulates strongly human perception and acquires fastest and numerously information [1]. The eye is an organ of sight to receive light reflected from objects in real world. The light rays are focused on the back of eyes to be converted to signal in order to be leaded to brain. The signals from light rays enable people to detect things in real world. The eyes do not only perceive objects according to their shapes on a plane but also detect their depths. The difference of phases of light rays coming to two eyes of a person from objects help people to cognize their depth [2].

Viewable angle formed by two lines from human eye to two points in space enables people to cognize the size and depth of objects. Two objects of the same size and the same distance to a person are perceived that they are same size because their viewable angles from eyes of the person to them are equal. Two objects of the same size placed at the different distances from a person are perceived the difference of their sizes because of the difference of their viewable angles from the person to them, the closer one is perceived the larger because its viewable angle is larger, on the contrary, the further the smaller because its viewable angle is smaller. Human eye can not perceive objects or detect details if viewable angle from the eye to them are so small. Human eye can detect a small detail if its viewable angle is enough big to separate the detail, this human possibility depends on the distance from the eye to the detail because viewable angle depends on the distance. The visional resolution is the capacity of human eye for detecting smallest details; it is the smallest viewable angle with which human eyes can perceive two different points in real world. The visional resolution depends on the physical ability of eyes of each person and the bright, colour, luminance of environment containing object [2].

For studying the human capacity of vision, Gestalt discovered that the acquired information in viewing the whole is more than acquired information in summing of components [1]. Accordingly, the data visualization is demanded to provide analysts with the way to view not only each variable but also all variables. In viewing the whole, analysts estimate not only the relation among the values of a variable but also the correlation among variables. The analysts' observation results in not only the cognition of data significance but also the possible discovery of new knowledge.

\subsection{The retinal variable}

Table 1. Retinal variables (according to Bertin $[3,4]$ )

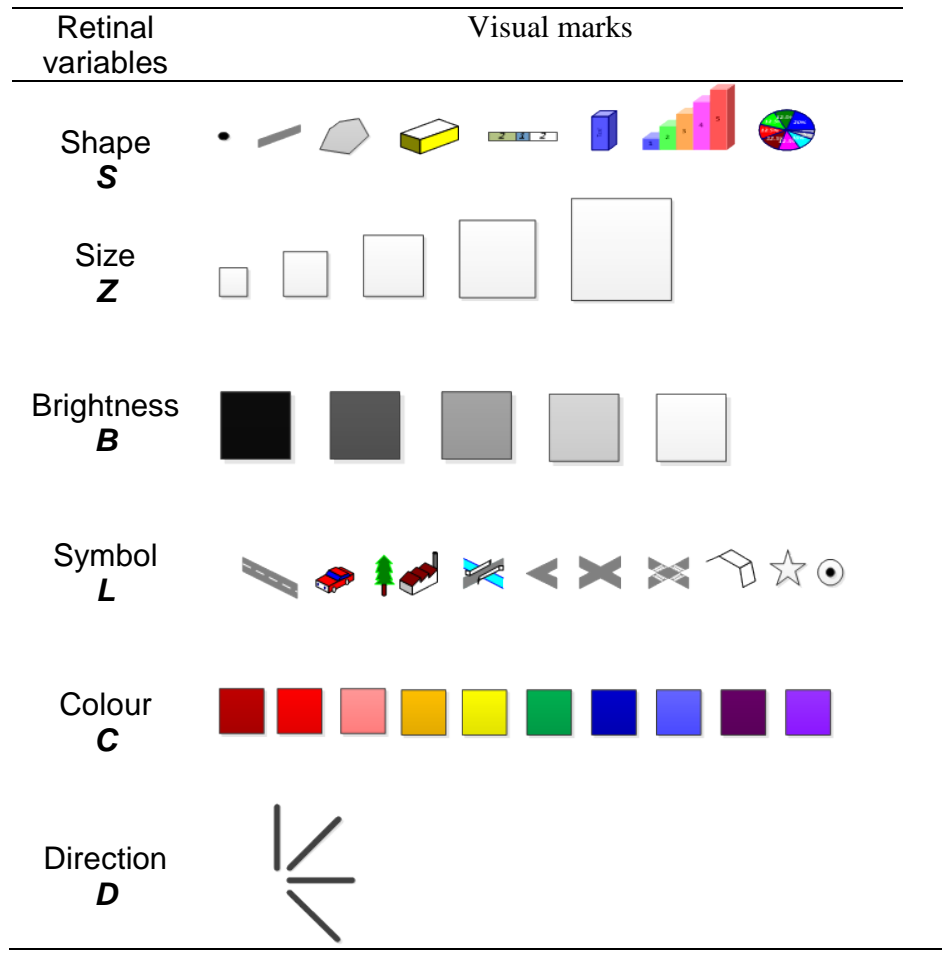

Data visualization refers to a mapping of data variables onto visual variables and data values onto marks displaying as graphs on planar environment, called screen. A visual variable representing a data variable is displayed 
as an axe, a visual variable representing data tuples of relational data variables is displayed as a coordinate system. The marks representing values of data or datatuples are shown as visual graphs at positions on axes or on coordinate systems. In other word, visual variables represent the values of data variables as graphs at positions on axes or on coordinate systems.

The visualization represents data as graphs positioned on 2D coordinate system of screen, and enhanced visual characteristics by retinal variables which consist of shapes, sizes, symbols, colours, brightness, directions (table 1) [3-5]. In addition to the six fundamental retinal variables, the graphic technique also provides with dynamic retinal variable such as flicker including frequency flicker and phase flicker [5]. Retinal variables suitable for the demands of visualization problem are chosen to integrate into visual variables, i.e. into graphs representing data variables.

\subsection{The visual feature}

The properties of visional perception and users' demands of visualization formulate visual features. The following visual features are utilized to study the effectiveness of a visual representation [3]:

Selective feature: The selective feature of visual variable refers to the perceptive isolation of different graphs representing different data variables.

Associative feature: The associative feature of visual variable refers to the perceptive groups of marks representing data values of the same category or the same attribute.

Ordered feature: The ordered feature of visual variable refers to the sequence of variables or values which can be arranged in perceptive order according to some particular property such as light vs. dark, and so on.

Quantitative feature: The quantitative feature of visual variable refers to the possibility perceiving the measure of the variation of data variable or the comparison between marks representing data values.

These properties are applied for enhancing the visual characteristics of visual graphs representing data set.

\section{The application of retinal variables for visualization}

In data visualization, retinal variables are integrated into graphs representing data variables to improve the visual characteristics of the graphs and increase the human capacity for perceiving information and acquiring knowledge from data. In several cases, it is not necessary to apply completely the four visual features mentioned above in a visual representation. The application of retinal variables for a visual graph representing data depends on the objective of visualization and /or users' demands.

In visualization of multivariable data, the selective and associate features are necessary for the isolation among graphs representing different variables in viewing comprehensively each graph, the quantitative feature is applied to detect higher and / or lower values, and the ordered feature is utilized to represent the time variable. A visual graph representing a data variable may be asked to meet one or more visual features. A visual graph is improved visual characteristics by applying a fundamental retinal variable as shape, size, brightness, symbol, colour, direction, and / or a product of some fundamental retinal variables.

The approaches to integrating retinal variables into visual graphs representing data variables are illustrated in this article with two data sets. The first illustration aims at the selection among graphs representing different data variables and the association of marks representing the values of the same data variable. The second not only meets the selective and associative features as the first, but also underlines the quantitative feature of particular marks representing data values.

\subsection{Integrating fundamental retinal variables into graphs representing data variables}

Let $G$ be a visual graph representing a data set comprising several componential graphs $G_{i}$, with $G=\left\{G_{i} \mid i=1,2, ..\right\}$, and $C=\left\{c_{j} \mid j=1,2, ..\right\}$ be the colour variable used as a fundamental retinal variable, where $c_{j}$ is a colour. Integrating the colour variable into the visual graph is to create the product of two sets $G$ and $C$, defined as follows.

$$
\begin{aligned}
& C \times G=\left\{c_{j} \mid j=1,2, . .\right\} \times\left\{G_{i} \mid i=1,2, . .\right\} \\
& C \times G=\left\{\left(c_{1}, G_{1}\right),\left(c_{2}, G_{2}\right), . .\right\}
\end{aligned}
$$

The product $C \times G$ is considered that different componential graphs are displayed with different colours, i.e. $i \neq i^{\prime} \Leftrightarrow j \neq j^{\prime}$. Accordingly, componential graphs are individually perceived because their colours are different. In other word, the product increases the selective feature of componential graphs representing different data variables and increases the associate feature of marks representing values of the same data variable. The number of colours is theoretically infinite, but the human possibility detecting different colours is limited. This property results in the finiteness of the number of colours utilized in the visualization of multivariable data.

\subsection{Integrating fundamental retinal variables into marks representing data values of a variable}

Let $G_{i}$, with $G_{i}=\left\{a_{n} \mid n=1,2, ..\right\} \quad$ be a componential graph representing a data variable, where each mark on the graph representing a quantitative value $a_{n}$ of the data 
variable. The graph $\boldsymbol{G}_{\bar{i}}$ was integrated with the colour variable $c_{i}$ to obtain $\left(c_{i}, G_{i}\right)$. The value $a_{n}$ of the data variable ranges from $a_{\min }$ to $a_{\max }$, i.e. $a_{n} \in\left[a_{\min }, a_{\max }\right]$. In some cases, the values need to be divided into groups of the same category and to be understood or perceived as value intervals .

Table 2. Wind speed in real number mapped onto wind force in natural number (according to http://www.vnbaolut.com/thientai bangcapgio.htm)

\begin{tabular}{ll}
\hline Wind speed $(\mathrm{m} / \mathrm{s})$ & Wind force \\
\hline $0.0-1.5$ & 1 \\
$1.5-3.3$ & 2 \\
$3.3-5.4$ & 3 \\
$5.4-7.9$ & 4 \\
$7.9-10.7$ & 5 \\
$10.7-13.8$ & 6 \\
$13.8-17.1$ & 7 \\
$17.1-20.7$ & 8 \\
$20.7-24.4$ & 9 \\
$24.4-28.4$ & 10 \\
$28.4-32.6$ & 11 \\
\hline
\end{tabular}

As an example, continuous real values of wind speed are converted to 11 levels of wind forces (Table 2). In visualization, the 11 different levels of wind force may be represented differently by the product of the brightness variable $B=\left\{b_{1}, b_{2}, \ldots, b_{11}\right\}$ and the colour variable of wind speed (Fig. 1).

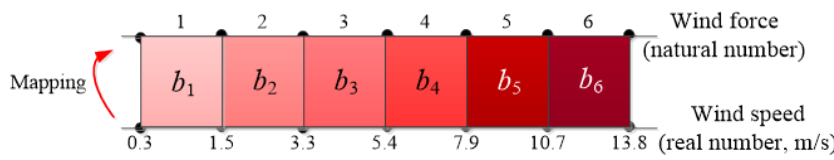

Figure 1. The product of brightness variable and colour variable to represent different levels of wind force.

\section{Multivariate cube integrated retinal variables to visually represent multivariable data}

\subsection{Multivariate cube}

Parallel coordinates consist of several parallel axes on a plane, where each axis shows the values of a data variable [6-9] (Fig. 2). For a data table, each data column is represented as an axis in parallel coordinates and each data tuple of the table is represent as a polyline connecting corresponding values on axes of the coordinates. The parallel coordinates enable user to answer elementary question [10] with the relation among data variables shown as the polylines representing data tuples. However, it is difficult to answer synoptic questions [10] because it does not show clearly the variation of a data variable as well as the correlation among different data variables. Indeed, taking a comprehensive view on an axis, viewer can not perceive the variation of the data variable which the axis represents. Meanwhile, the correlation among data variables may be cognized through polylines showing data tuples.

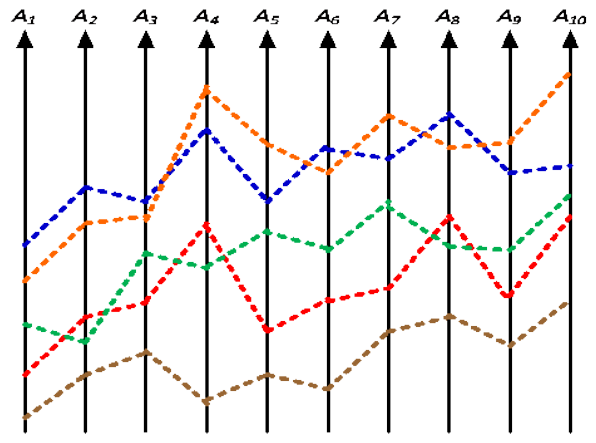

Figure 2. The parallel coordinates.

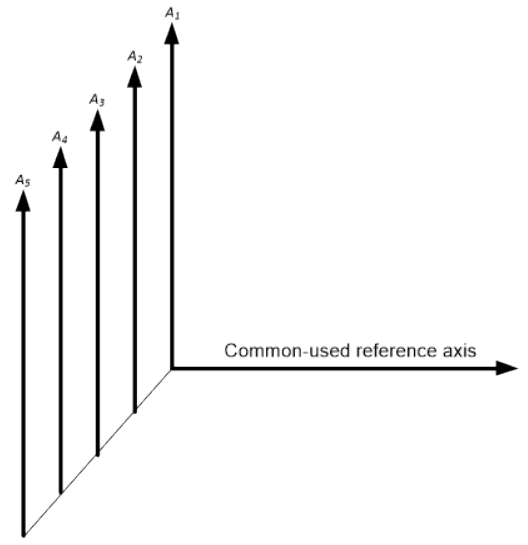

Figure 3. Multivariate cube modified from parallel coordinates

Multivariate cube is modified from parallel coordinates, where the common-used reference axis is rotated to the direction perpendicular to the plane of parallel coordinates [11, 12] (Fig. 3). In multivariate cube, each data variable is represented as an axis $A_{i} \mid \boldsymbol{i}=\mathbf{1}, 2, \ldots$ and referred to a common-used reference axis perpendicular to the axis $A_{i} \mid \boldsymbol{i}=1,2, \ldots$., where the common-used reference axis represents the variable on which all other variables depend. Multivariate cube enables user to answer elementary questions by viewing marks representing data values on graphs, to understand the variation of each data variable on the whole reference axis, and to cognize the correlation among data variables by viewing marks representing data values on the planes parallel with the plane of parallel coordinates and moved along to the common-used reference axis. 


\section{Some case studies applying multivariate cube integrated retinal variable}

\subsection{The evaluation of higher education curriculum on information system at Thu Dau Mot University}

Thu Dau Mot University (TDMU) is a provincial university located in Thu Dau Mot city belonging to Binhduong province which borders in the north on Hochiminh City, Vietnam. The TDMU provides diverse curricula for undergraduate and graduate students. It not only provides learners with academic knowledge but also trains them skill and attitude (Fig.4). Expected as a big center for higher education and a knowledge symbol of Binhduong province and Southeast Vietnam, the TDMU evaluates its results of education and research every year, where the data evaluating information system curriculum is used as a case study applying multivariate cube integrated retinal variable.

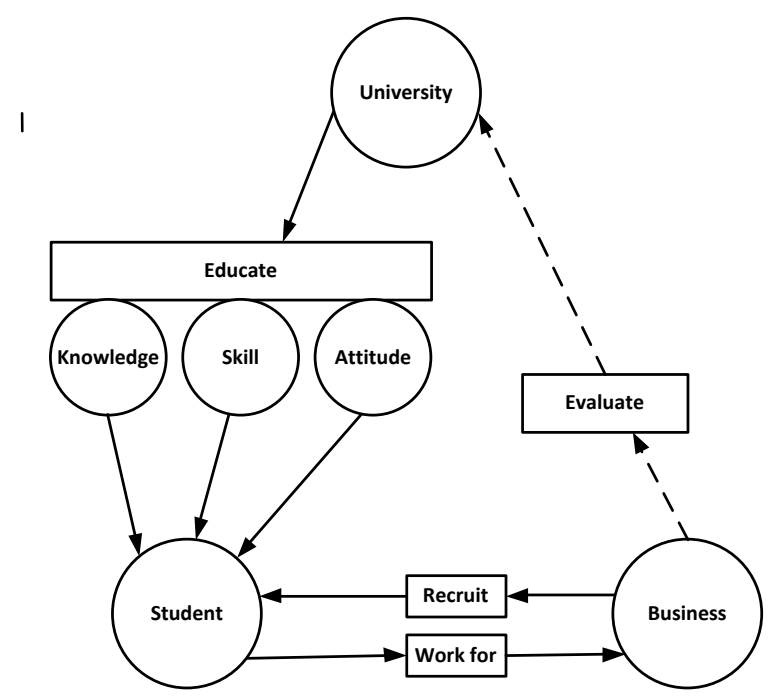

Figure 4. The triad of higher education "University Student - Business". The triad represents the relation between university, student, and business, where university educates students on knowledge, skill, and attitude to meet the recruitment need of business, meanwhile business recruits students graduated from university and evaluates the response rate of university with respect to its demand (Source: "Evaluating the competence of students graduated from information technology curricula in Hochiminh City." Master dissertation by Anh Van Thi Tran, 2013).

The evaluation is created on the statistical considerations concerning learning outcomes of the information system curriculum. Recruiters of business, alumni, last-year students, and lecturers are interviewed on their cognition and evaluation of the learning outcomes as well as their expectation of the students' achievement of learning outcomes. There are 18 learning outcomes applied for the survey, Knowledge of Basic Science (KBS), Knowledge of Basic Engineering (KBE), Knowledge of Advanced Engineering (KAE), Knowledge of Supplement (KOS), Capacity of Reasoning, Analyzing, and Solving Problems (RAS), Researching and Exploring Knowledge (REK), Thought of Systematic Level (TSL), Team Work (TWK), Communication (COM), Englishbased Communication (EBC), Society and Environment (SAE), Job Context and Business (JCB), Constituting Idea and Managing Technical System (MTS), Designing System of Information Technology (SIT), Implementation (IMP), Operating and Maintaining System of Information Technology (OAM), Attitude, Idea, and Education (AIE), Morality and Justice (MAJ). For the survey, almost one hundred interviewees rated each learning outcome at 5 levels and the values of following tables are mean.

Table 3. The statistics on the interviewees' cognition of the importance of learning outcomes (Source:

Survey of Tuyet Anh Thi Nguyen in a study funded by TDMU in 2016)

\begin{tabular}{lllll}
\hline $\begin{array}{l}\text { Learning } \\
\text { outcomes }\end{array}$ & $\begin{array}{l}\text { Recruiters } \\
(\mathrm{R})\end{array}$ & $\begin{array}{l}\text { Alumni } \\
(\mathrm{A})\end{array}$ & $\begin{array}{l}\text { Last- } \\
\text { year } \\
\text { students } \\
(\mathrm{S})\end{array}$ & $\begin{array}{l}\text { Lecturers } \\
(\mathrm{L})\end{array}$ \\
\hline KBS & 3 & 2.61 & 2.96 & 3.61 \\
KBE & 3.47 & 3.4 & 3.67 & 3.88 \\
KAE & 3.4 & 3.46 & 3.91 & 3.95 \\
KOS & 3.41 & 3.22 & 3.42 & 3.32 \\
RAS & 3.88 & 3.42 & 3.66 & 3.08 \\
REK & 3.69 & 3.53 & 3.02 & 3.66 \\
TSL & 3.88 & 2.97 & 3.09 & 3.55 \\
TWK & 3.88 & 4 & 3.37 & 3.76 \\
COM & 3.68 & 3.49 & 3.56 & 3.08 \\
EBC & 4.11 & 3.69 & 4.2 & 2.97 \\
SAE & 3.23 & 3.1 & 2.4 & 2.74 \\
JCB & 3.04 & 3.63 & 3.53 & 2.75 \\
MTS & 3.04 & 3.63 & 3.53 & 2.75 \\
SIT & 3.4 & 3.3 & 3.5 & 2.54 \\
IMP & 3.48 & 3.02 & 2.71 & 2.71 \\
OAM & 3.21 & 2.85 & 3.46 & 2.59 \\
AIE & 4.22 & 3.92 & 3.56 & 4.09 \\
MAJ & 3.93 & 3.71 & 3.39 & 3.88 \\
\hline
\end{tabular}

In visualization, the table 3 is studied as a data table with 5 variables and 18 data tuples. The data variables of recruiters $(R)$, alumni $(A)$, last-year students $(S)$, lecturers $(L)$ depend on the variable of learning outcomes. In other word, learning outcomes are studied as the common-used reference variable composed of 18 nominal and non- 
ordered values. The data table may be represented visually on a multivariate cube, where the axis of learning outcomes is shown as the common-used reference axis. The values of a variable are shown as marks on orthogonal coordinates formed by the common-used reference axis and the axis representing the variable. Accordingly, the data tables are displayed visually on four 2D orthogonal coordinates of parallel planes. The visual characteristics are enhanced with the integration of colour variable into the set of four variables, recruiters, alumni, last-year students, lecturers.

$$
\begin{aligned}
& C \times\{R, A, S, L\}=\left\{c_{1}, c_{2}, c_{3}, C_{4}\right\} \times\{R, A, S, L\} \\
& C \times\{R, A, S, L\}=\left\{\left(c_{1}, R\right),\left(c_{2}, A\right),\left(c_{3}, S\right),\left(c_{4}, L\right)\right\}
\end{aligned}
$$

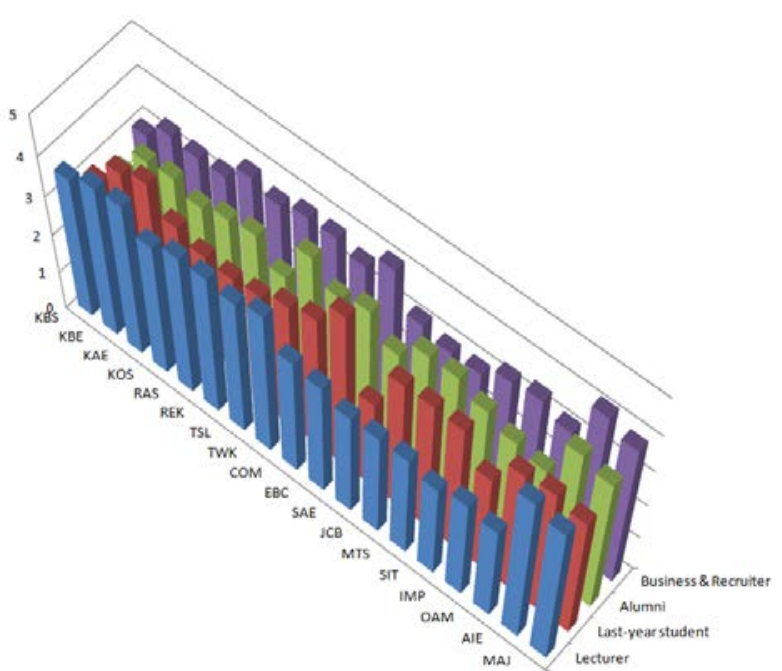

Figure 5. Multivariate cube integrated retinal variables to represent visually data of the table 3 on the interviewees' cognition of the importance of learning outcomes

Table 4. The statistics on the expectation of students' acquirement of learning outcomes (Source: Survey of Tuyet Anh Thi Nguyen in a study funded by TDMU in 2016)

\begin{tabular}{lllll}
\hline $\begin{array}{l}\text { Learning } \\
\text { outcomes }\end{array}$ & $\begin{array}{l}\text { Recruiters } \\
(\mathrm{R})\end{array}$ & $\begin{array}{l}\text { Alumni } \\
(\mathrm{A})\end{array}$ & $\begin{array}{l}\text { Last- } \\
\text { year } \\
\text { students } \\
(\mathrm{S})\end{array}$ & $\begin{array}{l}\text { Lecturers } \\
(\mathrm{L})\end{array}$ \\
\hline KBS & 4.141 & 3.694 & 3.866 & 4.305 \\
KBE & 4.141 & 3.694 & 3.866 & 4.305 \\
KAE & 4.313 & 4.019 & 4.081 & 4.583 \\
KOS & 4.177 & 3.944 & 3.657 & 4.068 \\
RAS & 4.281 & 4.083 & 3.614 & 4.234 \\
REK & 4.406 & 3.972 & 3.651 & 4.344 \\
TSL & 4.219 & 3.917 & 4.219 & 4.094 \\
TWK & 4.219 & 3.917 & 4.219 & 4.094 \\
\hline
\end{tabular}

\begin{tabular}{lllll}
\hline COM & 4.112 & 3.689 & 4.201 & 2.969 \\
EBC & 3.875 & 4.333 & 4.485 & 3.412 \\
SAE & 4 & 3.714 & 4.04 & 3.17 \\
JCB & 4.25 & 3.762 & 4.025 & 3.223 \\
MTS & 4.25 & 3.762 & 4.025 & 3.223 \\
SIT & 4.063 & 3.444 & 4.091 & 3.177 \\
IMP & 4.05 & 3.622 & 4.065 & 2.75 \\
OAM & 4.208 & 3.833 & 4.081 & 2.927 \\
AIE & 4.625 & 4.238 & 3.756 & 4.429 \\
MAJ & 4.6 & 4.378 & 3.847 & 4.463 \\
\hline
\end{tabular}

Looking at bars of the same colour, educational managers cognize the interviewees' awareness of the importance of each learning outcome, e.g. the alumni assess the knowledge of basic science at low level, the last-year students assess the society and environment at low level, and so on. Looking at bars of different colours on planes perpendicular to learning outcomes axis at different outcomes, educational mangers detect the learning outcomes assessed at low level by several interviewees, e.g. the Society and Environment is assessed at low level by almost interviewees, and so on.

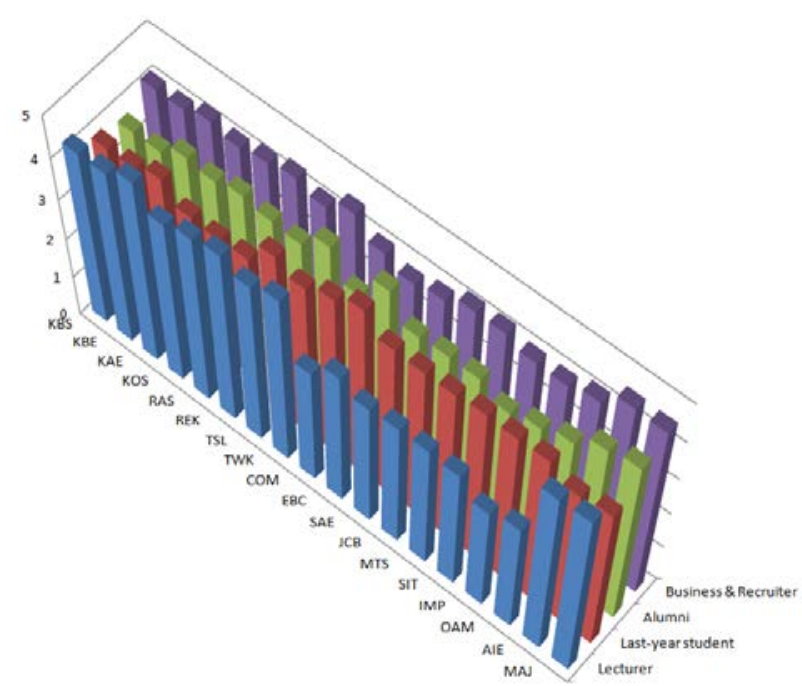

Figure 6. Multivariate cube integrated retinal variables to represent visually data of the table 4 on the interviewees' expectation of the students' acquirement of learning outcomes

Structured similarly to the table 3 , the table 4 provides with the interviewees' assessment of their expectation of students' achievement of learning outcomes. The data table 4 are represented visually on multivariate cube (Fig. 6) to enable educational managers to analyse students, possibility responding the demand of learning outcomes. The multivariate cube integrated retinal variables enables educational managers to detect learning outcomes of information system curriculum that the university needs to improve. In that, some learning outcomes should be understood by lecturers their importance. 


\subsection{The happenings of hand, foot, mouth disease in Binhduong province}

Binhduong province borders on northern Hochiminh City, Vietnam. It is about 2,700 square kilometers large with over 1.7 million habitants. Binhduong Medical Center for Providing Against Possible Contingencies is the governmental agency which is in charge of frequently keeping track of the epidemiologic situation over the whole province. In that, epidemiologic disease of hand, foot, mouth is also monitored. Data concerning the epidemiologic disease composed of the number of patients and the weather factors of rainfall, air humidity, and air temperature are recorded every week. In other words, each data variable as the patient number, rainfall, air humidity, and average temperature associates with the time variable. The data may be represented on a multivariate cube, where time axis is the common-used reference axis. The asked analytical questions are:

- What weather factors affect the number of patients?

- In which case, disease of hand, foot, mouth can happen?

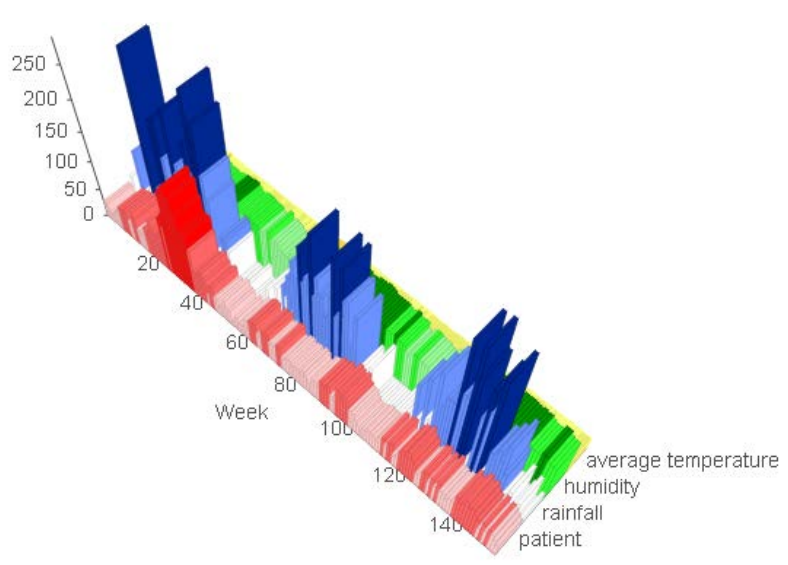

Figure 7. Multivariate cube integrated retinal variable to represent visually data concerning hand, foot, mouth disease in Binhduong province during 2012-2014.

The questions are answered with visual analytics. The case study utilizes data recorded by Binhduong Medical Center for Providing Against Possible Contingencies during 2012-2014. Multivariate cube represents data variables of the number of patients $(\boldsymbol{P})$, rainfall $(\boldsymbol{R})$, air average humidity $(\boldsymbol{H})$, and air average temperature $(\boldsymbol{D})$, which associate with time variable of week unit. The values of the variables are displayed as bars perpendicular to time axis. The two fundamental retinal variables are integrated into the componential graphs to enhance their visual characteristics.
The product of the colour variable $C=\{$ red,blue, green, yellow $\}$ and the set of data variables $V=\{P, R, H, D\}$ is displayed visually as the figure 7 with:

$$
\begin{aligned}
& C \times V=\{\text { red }, \text { blue }, \text { green, yellow }\} \times\{P . R, H, D\} \\
& C \times V=\{(\text { red }, P),(\text { blue }, R),(\text { green }, H),(\text { yellow }, D)\}
\end{aligned}
$$

The visual graph representing factors on multivariate cube integrated by colour variable (Fig. 7) provides analysts with the view on each colour to understand comprehensively the variation of each factor. However, two questions above have not been answered. It is necessary to stand out higher and lower values of each factor to find their specific relations. The value range of each factor is divided into three value levels.

The brightness variable will differentiate between higher and smaller values of each data variable when colour variable is integrated into data variables, including the number of patients and weather factors. The chosen brightness variable $B=\left\{b_{1}, b_{2}, b_{3}\right\}$ is integrated into the colour variable $\quad C=\{$ red, blue, green, yellow $\}$ corresponding to three value levels, the dark colour for highest values, the light for lowest values, and the medium brightness for other values (Fig. 7).

The difference of colours among data variables and colour brightness of each variable enhance the human possibility in perceiving the happenings of hand, foot, mouth disease in Binhduong province. For the case study (Fig. 7), viewing on the array of red bars, analyst easily cognizes that the number of patients of hand, foot, mouth disease in Binhduong province changes periodically over time. The bars of dark colours of the number of patients and the factors of rainfall and humidity show the correlation between the number of patients and the factors whereas average temperature does not relate perceptibly to the development of the epidemic. The periodicity of data and the correlation of data variables enable epidemiologic analysts to detect the natural law of the development of hand, foot, mouth disease by answering two questions above.

\section{Conclusion}

Multivariate cube is modified from parallel coordinates with the common-used reference axis is rotated to the direction perpendicular to the plane of the parallel coordinates. Data variables are shown visually on parallel planes containing the common-used reference axis to constitute a multivariate cube. Each plane displays visually the values of a data variable. Multivariate cube is applied illustratively for the data concerning the evaluation of learning outcomes of information system curriculum at Thu Dau Mot University, surveyed in 2016, and the data relating to hand, foot, and mouth disease in Binhduong province during 2012-2014.

Multivariate cube integrated retinal variable enhances the visual characteristics of the cube. The article presented two approaches to using retinal variable to enhance visual 
features of graphs representing data variables. The first is the integration of a retinal variable into data variables, each of which associates with an element of the retinal variable to enhance the selective and associative features. The second is the integration of a retinal variable into data intervals divided to stand out specific values. Two approaches to integrating retinal variable into data variable enable analysts to answer not only elementary but also synoptic questions. For responding synoptic questions, analysts may understand perceptively the variation of data while viewing comprehensively visual graphs and may discover natural law, e.g. its periodicity, and understand perceptively the weather factors affecting the disease happenings while analyzing visually the correlation among data variables.

The graphic techniques provide users with soft tools to control flexibly diverse types of display suitable for visual analysis. The soft tools of selection, show / hide, zoom-in / zoom-out, window, rotation enable users to make good the limitation of screen and human capacity of vision. With soft tools of graphic technique, user can change retinal variable while integrating it into graphs and change the display to acquire information and knowledge as far as possible.

\section{References}

[1] D. S. Alexandre and J. M. R. S. Tavares, "Introduction of Human Perception in Visualization," International Journal of Imaging and Robotics ${ }^{\mathrm{TM}}$, vol. 4, pp. 60-70, 2010.

[2] A. Dix, J. Finlay, G. D. Abowd, and R. Beale, HumanComputer Interaction: Springer US, 2005.

[3] J. Bertin, "General Theory, from Semiology of Graphics," in The Map Reader. Theories of Mapping Practice and Cartographic Representation, M. Dodge, R. Kitchin, and C. Perkins, Eds., ed: John Wiley \& Sons, Ltd., 2011, pp. 8-16.

[4] J. Bertin, Semiology of Graphics, 1983.

[5] M. Green, "Toward a Perceptual Science of Multidimensional Data Visualization: Bertin and Beyond," ERGO/GERO Human Factors Science, Citeseer, 1998.

[6] A. Inselberg, Parallel Coordinates: Visual Multidimensional Geometry and Its Applications. 233 Spring Street, New York, NY 10013, USA: Springer Dordrecht Heidelberg London New York, 2008.

[7] G. Andrienko and N. Andrienko, "Constructing Parallel Coordinates Plot for Problem Solving," presented at the Smart Graphics '01, Hawthorne, NY, USA, 2001.

[8] A. Inselberg and B. Dimsdale, "Parallel Coordinates: a Tool for Visualizing Multi- Dimensional Geometry," IEEE, pp. 361-378, 1990.

[9] A. Inselberg, "The plane with parallel coordinates," The Visual Computer, vol. 1, pp. 69-91, 1985.

[10] N. Andrienko, G. Andrienko, N. Pelekis, and S. Spaccapietra, "Basic concepts of movement data," in Mobility, Data Mining and Privacy, Geographic Knowledge Discovery, F. Giannotti and D. Pedreschi, Eds., ed Berlin Heidelberg: Springer-Verlag 2008, pp. 15-38.
[11] H. T. Nguyen, A. V. T. Tran, T. A. T. Nguyen, L. T. Vo, and P. V. Tran, "Multivariate Cube for Representing Multivariable Data in Visual Analytics," in Context-Aware Systems and Applications, Thu Dau Mot, Viet Nam, 2016, pp. 91-100.

[12] H. T. Nguyen, T. V. Tran, P. V. Tran, and H. Dang, "Multivariate Cube for Visualization of Weather Data," presented at the IEEE 2013 International Conference on Control, Automation and Information Science, ICCAIS 2013, Nha Trang, Vietnam, 2013. 